\title{
Remote Plasma Maintenance in Low-Pressure Discharges with an External Magnetic Field
}

\author{
Stiliyan Lishev ${ }^{1}$, Antonia Shivarova ${ }^{1}$, Khristo Tarnev ${ }^{2}$ \\ ${ }^{1}$ Faculty of Physics, Sofia University, Sofia, Bulgaria \\ ${ }^{2}$ Department of Applied Physics, Technical University-Sofia, Sofia, Bulgaria \\ Email: ashiva@phys.uni-sofia.bg
}

Received August 18, 2012; revised September 16, 2012; accepted September 24, 2012

\begin{abstract}
The spatial structure of remote plasma regions in rf discharges is analyzed based on a 2D model of free-fall regime discharge maintenance. Since the study is directed towards description of the magnetic filter region in the tandem plasma sources of negative hydrogen ions, hydrogen discharges are considered, with a weak magnetic field located outside the region of the rf power deposition. With the formation of different regions in the discharge - the rf power deposition region, the region with electron magnetization, the transition between them and the region behind the filter-the results display superimposed effects of nonlocal discharge maintenance without and with a magnetic field. Slight decrease of the electron temperature accompanied with strong drop of the electron density is the "pure" effect of the plasma expansion in regions without external magnetic field. Strong drop of the electron temperature accompanied with formation of a maximum of the electron density in the filter region is the "pure" effect of the plasma expansion through a magnetic field. Based on the results for the spatial distribution of the electron density and temperature obtained with shifting the position of the magnetic filter, optimization of the source regarding high yield of volume-produced negative ions is discussed.
\end{abstract}

Keywords: Remote Plasma Maintenance; Tandem Plasma Sources; Magnetic Filter; Hydrogen Discharges; Free-Fall Regime

\section{Introduction}

The construction of the rf sources for plasma processing technology [1-3] has brought to the fore the nonlocality of the discharge behavior and the mechanisms of the remote plasma maintenance. Concerning hydrogen discharges, the rf source of negative hydrogen ion beams [4] developed for the neutral-beam-injection plasma heating in the international tokamak ITER is a particular case of such type of a discharge. Since the source is a twochamber one with rf power deposition to the first chamber and plasma expansion in the second-bigger sizechamber, effects of nonlocality, i.e. the fluxes (chargedparticle and electron-energy fluxes), govern its operation [5]. Moreover, with its design of a tandem source with a magnetic filter located in the second chamber, the plasma expanding from the first chamber passes through a region with a magnetic field. In a way, the description of the source operation provides a possibility for studying combined effects of nonlocal discharge behavior both without and with an external magnetic field, as it is done here.

Spatial separation, by a magnetic filter for electron cooling, of two regions in the discharge with high- and low-energetic electrons (respectively, with high and low electron temperature) is in the basis of the idea for the tandem source [6] developed, starting in the 80 's of the last century, initially for dc (filament) sources of negative hydrogen ions. The idea stems from a conclusion that such a configuration of the source ensures optimum conditions for the two-step reaction $-\mathrm{e}+\mathrm{H}_{2} \rightarrow \mathrm{H}_{2}(v)+\mathrm{e}$, $\mathrm{e}+\mathrm{H}_{2}(v) \rightarrow \mathrm{H}^{-}+\mathrm{H}$-for volume production of the negative ions $\left(\mathrm{H}^{-}\right)$via dissociative attachment of electrons to highly vibrationally excited molecules $\mathrm{H}_{2}(v)$ : vibrational excitation of the molecules in the region with a high electron temperature and negative ion production in the region with a low electron temperature.

The operation of the magnetic filter as an electron cooler has been usually stressed on as a result from the source modeling [7-15], involving transport processes acting in a combination with collisions. Emphasizing the role of the transport processes described within the fluid plasma theory, the 1D-, 2D- and 3D-models in [16] provide detailed description of the filter operation showing both lowering of the electron temperature and formation of a maximum of the electron density in the filter region. Reduced - by the magnetic field - thermal conductivity, i.e. suppressed nonlocality in the electron heating, acting 
together with diamagnetic drifts is shown to cause the drop of the electron temperature in the filter region and the formation of a groove there. Diffusion and thermal diffusion acting together appear to be responsible for the formation of the maximum of the electron density in the filter region, slightly shifted from the source axis due to an $(\mathbf{E} \times \mathbf{B})$-drift. The main trends in this pattern of the filter operation have been recently confirmed by both modeling based on PIC and fluid-plasma-model simulations $[17,18]$ and experiments $[19,20]$ on the electronegativity and the concentration of the negative hydrogen ions. The latter shows that the spatial distribution of the electron density and temperature, and of the plasma potential, stemming from the nonlocal discharge behavior determines the conditions for an effective production of the ions. This initiates the theoretical analysis presented here on the changes in the spatial distribution of the plasma parameters with changing the position of the magnetic filter.

The spatial structure of remote plasmas sustained without and with an external magnetic field is discussed in the study. Hydrogen discharges in a free-fall regime are considered within a 2D fluid-plasma model regarding description of the magnetic filter region in the rf tandem plasma sources. The results outline the effects of nonlocality in discharges with a localized rf power deposition and plasma expansion into a region with an external magnetic field. The transition between the power-deposition and magnetic-filter regions as well as the region behind the filter displays the effects of plasma expansion without magnetic field: slight decrease of the electron temperature accompanied with strong drop of the electron density. Strong drop of the electron temperature and formation of a maximum of the electron density in the filter region are the characteristics of the plasma expansion through a magnetic field. The results obtained for the distribution of the electron density and temperature are discussed regarding optimization of the source with respect to high yield of volume-produced negative ions.

\section{Basis of the Model}

The model presented here is an extension of the 2D model from [16] towards description of the free-fall regime maintenance of hydrogen discharges in an external magnetic field.

The configuration of the discharge vessel is schematically shown in Figure 1(a). The rf power deposition applied for the discharge production is localized (Figure 1(b)), with a half of a super-Gaussian profile in the $z$ direction:

$$
P_{\mathrm{w}}(z)=P_{\mathrm{w} 0} \exp \left[-\frac{1}{2}\left(\frac{z}{\sigma_{\mathrm{p}}}\right)^{2 m}\right]
$$

where $P_{\mathrm{w} 0}$ is its maximum at $z=0$ and $\sigma_{\mathrm{p}}$ scales its width. The magnetic field - the field of the magnetic filter - is also localized, positioned behind the region of the $\mathrm{rf}$ power deposition. Directed along the $y$-axis, the field is homogeneous in the $x$-direction, with a Gaussian profile in the $z$-direction centered at $z=z_{0}$ :

$$
B(z)=B_{0} \exp \left[-\frac{\left(z-z_{0}\right)^{2}}{2 \sigma_{B}^{2}}\right]
$$

where $B_{0}=B\left(z=z_{0}\right)$ and $\sigma_{B}$ scales its width. In the presentation of the results in the next section, the position $z_{0}$ has been varied.

The results given further on are for the distribution of the plasma parameters in the $(x-z)$-plane (Figure 1(a)), i.e. perpendicularly to the filter field. The species in the discharge are electrons, the three types of positive ions $\left(\mathrm{H}^{+}, \mathrm{H}_{2}^{+}\right.$and $\left.\mathrm{H}_{3}^{+}\right)$and hydrogen atoms $(\mathrm{H})$ and molecules $\left(\mathrm{H}_{2}\right)$. The rate coefficients of the elementary processes are calculated with a Maxwellian velocity distribution assumed for the electrons. The magnetic field is weak, so the electrons are magnetized and the ions are left unmagnetized. The gas pressure is low and the discharge maintenance is in the free-fall regime. The latter is specified by accounting for the nonlinear inertia term and for the inelastic collisions for particle production in the momentum equations of the positive ions. The elementary processes for production and destruction of charged particles and hydrogen atoms are listed in Table 1; the references for the corresponding rate coefficients are also given in the table.

The equations describing the electron component are the continuity equation and the electron energy balance equation:

$$
\begin{gathered}
\frac{\partial n_{\mathrm{e}}}{\partial t}+\nabla \cdot \boldsymbol{\Gamma}_{\mathrm{e}}=\frac{\delta n_{\mathrm{e}}}{\delta t} \\
\frac{3}{2} \frac{\partial\left(n_{\mathrm{e}} T_{\mathrm{e}}\right)}{\partial t}+\nabla \cdot \mathbf{J}_{\mathrm{e}}=P_{\mathrm{w}}-P_{\mathrm{coll}}-\mathrm{e} \boldsymbol{\Gamma}_{\mathrm{e}} \cdot \mathbf{E}_{\mathrm{dc}}
\end{gathered}
$$

where $n_{\mathrm{e}}, T_{\mathrm{e}}$ and $\mathbf{E}_{\mathrm{dc}}$ are, respectively, the electron density and temperature (in energy units) and the dc field formed in the discharge $\left(\mathbf{E}_{\mathrm{dc}}=-\nabla \Phi\right)$; $\mathrm{e}$ is the elementary charge.

The $x$ - and $z$-components

$$
\begin{aligned}
\Gamma_{\mathrm{e}(x, z)}= & b_{\perp \mathrm{e}} n_{\mathrm{e}} \partial_{(x, z)} \Phi-D_{\perp \mathrm{e}} \partial_{(x, z)} n_{\mathrm{e}}-D_{\perp \mathrm{e}}^{T} \frac{n_{\mathrm{e}}}{T_{\mathrm{e}}} \partial_{(x, z)} T_{\mathrm{e}} \\
& \pm b_{\mathrm{de}} n_{\mathrm{e}} \partial_{(z, x)} \Phi \mp D_{\mathrm{de}} \partial_{(z, x)} n_{\mathrm{e}} \\
& \mp D_{\mathrm{de}}^{T} \frac{n_{\mathrm{e}}}{T_{\mathrm{e}}} \partial_{(z, x)} T_{\mathrm{e}}
\end{aligned}
$$

of the electron flux $\boldsymbol{\Gamma}_{\mathrm{e}}$ account for electron mobility, diffusion and thermal diffusion across the magnetic field 
(respectively, the first three terms in the right-hand side of (5)), for $(\mathbf{E} \times \mathbf{B})$-drift (the fourth term) and for diamagnetic drifts due to density and temperature gradients (the last two terms). The corresponding coefficients are $b_{\perp \mathrm{e}}=b_{\| \mathrm{e}} /\left[1+\left(\Omega_{\mathrm{e}} / v_{\mathrm{e}}\right)^{2}\right]$, $D_{\perp \mathrm{e}} \equiv D_{\perp \mathrm{e}}^{T}=D_{\| \mathrm{e}} /\left[1+\left(\Omega_{\mathrm{e}} / v_{\mathrm{e}}\right)^{2}\right], b_{\mathrm{de}}=\left(\Omega_{\mathrm{e}} / v_{\mathrm{e}}\right) b_{\perp \mathrm{e}}$ and $D_{\mathrm{de}} \equiv D_{\mathrm{de}}^{T}=\left(\Omega_{\mathrm{e}} / v_{\mathrm{e}}\right) D_{\perp \mathrm{e}}$; here $b_{\| \mathrm{e}}=\mathrm{e} / m_{\mathrm{e}} v_{\mathrm{e}}$ and $D_{\| \mathrm{e}}=T_{\mathrm{e}} / m_{\mathrm{e}} v_{\mathrm{e}}$ are the mobility and diffusion coefficients along the magnetic field, $\Omega_{\mathrm{e}}=\mathrm{e} B / m_{\mathrm{e}}$ is the electron gyrofrequency (with $m_{\mathrm{e}}$ being the electron mass) and $v_{\mathrm{e}}=v_{\mathrm{e}-\mathrm{a}}+v_{\mathrm{e}-\mathrm{m}}$ is the frequency of elastic collisions with atoms and molecules. The electron production and losses are via the processes numbered by "1", "5" and " 6 " in Table 1.

The $x$ - and $z$-components

$$
J_{\mathrm{e}(x, z)}=-\chi_{\perp \mathrm{e}} \partial_{(x, z)} T_{\mathrm{e}} \mp \chi_{\mathrm{de}} \partial_{(z, x)} T_{\mathrm{e}}+\frac{5}{2} T_{\mathrm{e}} \Gamma_{\mathrm{e}(x, z)}
$$

of the electron energy flux $\mathbf{J}_{\mathrm{e}}$ include the conductive (the first two terms in the right hand side) and convective (the last term) fluxes. The corresponding components of the thermal conductivity tensor are

$\chi_{\perp \mathrm{e}}=\chi_{\| \mathrm{e}} /\left[1+\left(\Omega_{\mathrm{e}} / v_{\mathrm{e}}\right)^{2}\right]$ and $\chi_{\mathrm{de}}=\left(\Omega_{\mathrm{e}} / v_{\mathrm{e}}\right) \chi_{\perp \mathrm{e}}$ with $\chi_{\| \mathrm{e}}=(5 / 2) n_{\mathrm{e}} D_{\| \mathrm{e}}$. The electron energy losses in collisions $P_{\text {coll }}$ accounted for are the same as in $[16,24]$ : atom excitation and ionization, dissociation, ionization, excitation of vibrational and singlet states of the molecules and elastic collisions of electrons with atoms and molecules. The last term in the right-hand side of (4) accounts for electron energy losses for maintenance of the dc field in the discharge.

The equations describing the ion plasma components are the continuity equations and the momentum equations

$$
\begin{gathered}
\frac{\partial n_{j}}{\partial t}+\nabla \cdot \boldsymbol{\Gamma}_{j}=\frac{\delta n_{j}}{\delta t} \\
m_{j}\left(\mathbf{v}_{j} \cdot \nabla\right) \mathbf{v}_{j}=\mathrm{e} \mathbf{E}_{\mathrm{dc}}-\left(\mu_{j} v_{j}\right) \mathbf{v}_{j}
\end{gathered}
$$

of the three types of positive ions $(j=1,2,3$, respectively, for the $\mathrm{H}^{+}-, \mathrm{H}_{2}^{+}$- and $\mathrm{H}_{3}^{+}$-ions), the latter written in a stationary form. Here $n_{j}, \mathbf{v}_{j}$ and $m_{j}$ are the corresponding densities, velocities and masses and $\left(\delta n_{j} / \delta t\right)$ is the ion production and losses in inelastic collisions, as specified by the processes in Table 1.

Since a free-fall regime of discharge maintenance is considered the nonlinear inertia terms in the momentum equations of the ions (the terms in the left-hand side of (8)) are taken into account as dominating over the diffusion. Also, due to the reduction of the elastic collision frequency at low gas pressure, the inelastic collisions for
Table 1. Processes involved in the charged particle balance and the balance of the hydrogen atoms.

\begin{tabular}{ccc}
\hline $\begin{array}{c}\text { Reaction } \\
\text { number }\end{array}$ & Process & Reference \\
\hline 1 & $\mathrm{e}+\mathrm{H}_{2}\left(\mathrm{X}^{1} \Sigma_{\mathrm{g}}^{+}\right) \rightarrow \mathrm{e}+\mathrm{H}_{2}^{+}(v)+\mathrm{e}$ & {$[21]$} \\
2 & $\mathrm{e}+\mathrm{H}_{2}\left(\mathrm{X}^{1} \Sigma_{\mathrm{g}}^{+}\right) \rightarrow \mathrm{e}+\mathrm{H}(1 \mathrm{~s})+\mathrm{H}(1 \mathrm{~s})$ & {$[21]$} \\
3 & $\mathrm{e}+\mathrm{H}_{2}^{+}(v) \rightarrow \mathrm{e}+\mathrm{H}^{+}+\mathrm{H}(1 \mathrm{~s})$ & {$[21]$} \\
4 & $\mathrm{H}_{2}^{+}+\mathrm{H}_{2} \rightarrow \mathrm{H}_{3}^{+}+\mathrm{H}$ & {$[22]$} \\
5 & $\mathrm{e}+\mathrm{H}(1 \mathrm{~s}) \rightarrow \mathrm{e}+\mathrm{H}^{+}+\mathrm{e}$ & {$[21]$} \\
6 & $\mathrm{e}+\mathrm{H}_{3}^{+} \rightarrow \mathrm{H}+\mathrm{H}^{+} \mathrm{H}$ & {$[21]$} \\
7 & $\mathrm{e}+\mathrm{H}_{3}^{+} \rightarrow \mathrm{e}+\mathrm{H}^{+}+2 \mathrm{H}$ & {$[21]$} \\
8 & $\mathrm{H}+\mathrm{H}_{\mathrm{ad}} \rightarrow \mathrm{H}_{2}$ & {$[23]$} \\
\hline
\end{tabular}

ion production $\left(\delta n_{j}^{(i)} / \delta t\right)$ are included in (8) via the notation

$$
\mu_{j} v_{j}=\sum_{l=1}^{2} \mu_{j l} v_{j l}+\frac{m_{j}}{n_{j}} \frac{\delta n_{j}^{(i)}}{\delta t}
$$

which combines both elastic collisions with atoms and molecules (respectively, $l=1,2$ ), with reduced masses $\mu_{j l}$ and frequencies $v_{j l}$, and inelastic collisions (the second term in the right-hand side of (9)) for production of the ions.

The inertia term specifying low gas-pressure discharges, respectively, free-fall regime discharge maintenance, shows evidence in the wall sheath [25] where the dc potential drops strongly. In general, the inertia term plays the role of a retarding force that limits the strong increase of the velocity in the wall sheath. Since in the wall sheath the dc electric field is almost perpendicular to the corresponding wall, the parallel- to that wallcomponent of the ion velocity can be neglected ([25] where it is shown that this does not influence the accuracy of the solution) and, thus, (8) can be written as:

$$
\begin{aligned}
& m_{j} v_{j x} \frac{\partial v_{j x}}{\partial x}=-\mathrm{e} \frac{\partial \Phi}{\partial x}-\left(\mu_{j} v_{j}\right) v_{j x} \\
& m_{j} v_{j z} \frac{\partial v_{j z}}{\partial z}=-\mathrm{e} \frac{\partial \Phi}{\partial z}-\left(\mu_{j} v_{j}\right) v_{j z} .
\end{aligned}
$$

Further on the collisionless-case energy-conservation law of the ions

$$
\frac{1}{2} m_{j} v_{j(x, z)}^{2}=\mathrm{e}\left(\Phi_{m}-\Phi\right)
$$

can be used for determination of the spatial derivatives of the velocity components $\left(\partial v_{j x} / \partial x\right.$ and $\left.\partial v_{j x} / \partial x\right)$ in (10); $\Phi_{m}$ is the maximum value of the potential. This permits reaching - in an explicit form - solutions for the ion velocity components. 


$$
\begin{gathered}
v_{j x}=\frac{\mathrm{e} \partial_{x} \Phi}{\sqrt{\frac{\mathrm{e} m_{j}}{2}} \frac{\partial_{x} \Phi}{\sqrt{\Phi_{m}-\Phi}}-\left(\mu_{j} v_{j}\right)} \\
v_{j z}=\frac{\mathrm{e} \partial_{z} \Phi}{\sqrt{\frac{\mathrm{e} m_{j}}{2}} \frac{\partial_{z} \Phi}{\sqrt{\Phi_{m}-\Phi}}-\left(\mu_{j} v_{j}\right)}
\end{gathered}
$$

needed for completing the ion fluxes $\Gamma_{j}$ in (7). In a way, the two retarding forces - the inertia term and the momentum losses in collisions - are combined defining effective mobilities specified for each direction.

The Poisson equation

$$
\Delta \Phi=\frac{\mathrm{e}}{\varepsilon_{0}}\left(n_{\mathrm{e}}-\sum_{j=1}^{3} n_{j}\right),
$$

the balance of the hydrogen atoms

$$
\frac{\partial N_{a}}{\partial t}-\operatorname{div}\left(D_{a} \nabla N_{a}\right)=\frac{\delta N_{a}}{\delta t}
$$

and the expression for the gas pressure

$$
p=\kappa T_{g}\left(N_{a}+N_{m}\right)
$$

complete the initial set of equations. In (13)-(15), $\varepsilon_{0}$ is the vacuum permittivity, $T_{g}$ is the gas temperature, $\kappa$ is the Boltzmann constant, $N_{\mathrm{a}}$ and $N_{\mathrm{m}}$ are the densities of the hydrogen atoms and molecules and $D_{\mathrm{a}}$ is the diffusion coefficient of the hydrogen atoms determined according to [26].

Like in [16], the boundary conditions are for the fluxes at the walls (the fluxes of the charged particles and the electron energy flux) and for a zero potential of the dc electric field (due to the metal walls of the discharge vessel).

\section{Results and Discussions}

The results discussed here are for the axial $(z)$ variation at the discharge axis $(x=0)$ of the plasma parameters obtained from the 2D-model description presented in the previous section. The size of the modeling domain is $L_{z}=20 \mathrm{~cm}$ and $L_{x}=10 \mathrm{~cm}$ (Figure 1). Both the position $z_{0}$ of the center of the magnetic filter and the gas pressure has been varied, respectively, in the ranges $z_{0}=$ $(10-20) \mathrm{cm}$ and $p=(3.5-10)$ mTorr. The magnetic field in its maximum is $B_{0}=50 \mathrm{G}$ with $\sigma_{B}=1.58 \mathrm{~cm}$ and $m=2$; the extension in the $z$-direction of the power deposition is given by the value of $\sigma_{\mathrm{p}}=4.73 \mathrm{~cm}$. The total applied power is $100 \mathrm{~W}$. The value of the gas pressure assumed is $300 \mathrm{~K}$.

\subsection{Discharge Structure and Its Changes with Varying the Position of the Magnetic Field}

Figures 2 and $\mathbf{3}$ present the results for the electron tem-

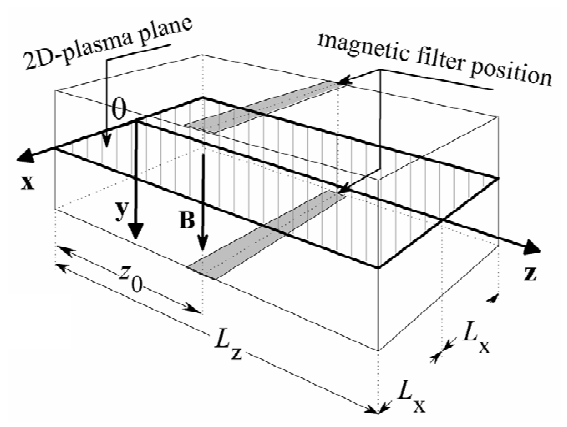

(a)



(b)

Figure 1. Configuration of the plasma volume (a) and illustration of the $z$-variation of the filter field and of the $\mathbf{r f}$ power input (b).



(a)

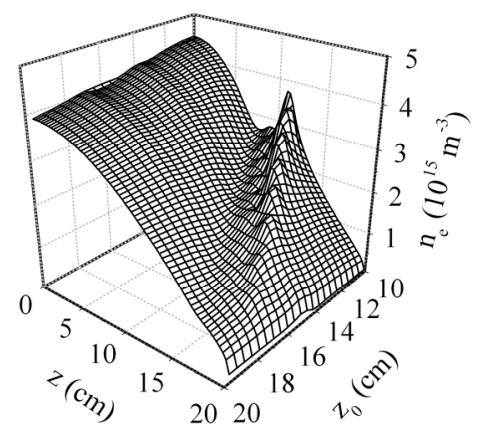

(b)

Figure 2. Changes in the axial variation (at $x=0$ ) of the electron temperature (a) and density (b) with varying the position $z_{0}$ of the maximum of the magnetic field; $p=4$ mTorr.

perature $T_{\mathrm{e}}$ and density $n_{\mathrm{e}}$ for $p=4 \mathrm{mTorr}$ and varying $z_{0}$. 


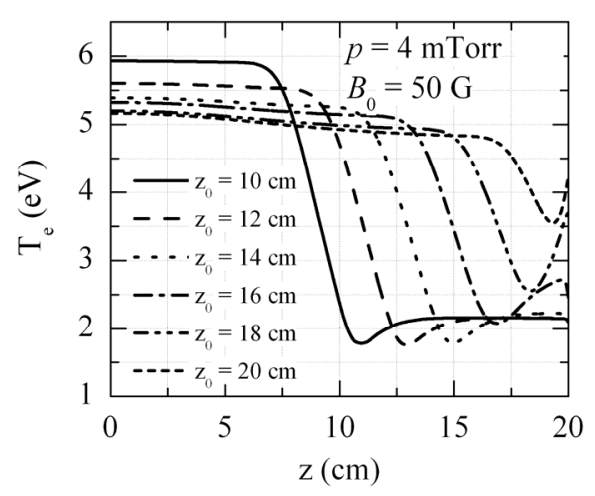

(a)

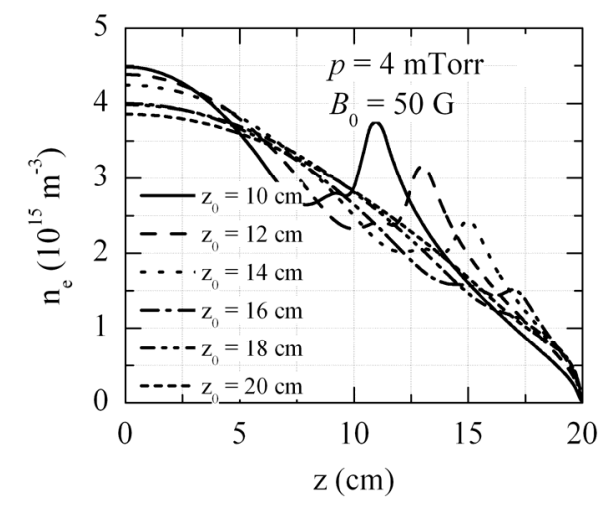

(b)

Figure 3. Comparison of the axial variation (at $x=0$ ) of the electron temperature (a) and density (b) for different values of $z_{0}$, as marked on the figure; $p=4 \mathrm{mTorr}$.

With the extension of the power deposition region of about $\Delta z_{\mathrm{p}} \approx 5 \mathrm{~cm}$ and of the magnetic filter region of about $\Delta z_{B} \approx 4 \mathrm{~cm}$, shifting the position $z_{0}$ of the maximum of the magnetic field between $10 \mathrm{~cm}$ and 20 $\mathrm{cm}$ displays effects of plasma expansion from the power deposition region into regions without and with an external magnetic field. The axial profiles of the plasma parameters (Figures 2 and 3) obtained for $z_{0} \approx 15 \mathrm{~cm}$ show the complete pattern of consecutively ordered regions in the discharge: plasma expansion from the power deposition region first in a region without a magnetic field followed by plasma expansion through the magnetic field and ending with expansion again in a region without a magnetic field. Thus, the nonlocality governing the operation of the discharge via the electron-energy and charged-particle fluxes from the power deposition region is the factor determining the axial variations of the electron density and temperature.

In the rf power deposition region the electron temperature stays almost constant and the electron density slightly decreases.

The drastic changes in the axial profiles of $T_{\mathrm{e}}$ and $n_{\mathrm{e}}$ are within the full width $\Delta z_{B} \approx 4 \mathrm{~cm}$ of the magnetic filter: strong drop of $T_{\mathrm{e}}$, i.e. an effect of electron cooling by the magnetic field, and formation of a maximum of $n_{\mathrm{e}}$ in the region of the electron magnetization. The physical pattern which stays behind the changes in the spatial distribution of the plasma parameters in the filter region has been described in details in [16]. As a summary it is, as follows.

The strong drop of $T_{\mathrm{e}}$ in the filter region is due to reduced - by the magnetic field - nonlocality of the electron heating. With the localized rf power applied, the plasma existence outside the region of its deposition is due to the electron energy flux (and charged particle fluxes) from this region.

The suppression - by the filter field - of the coefficient $\chi_{\perp \mathrm{e}}$ of thermal conductivity, reduces the electron energy flux $\mathbf{J}_{\mathrm{e}}$ and causes the drop of $T_{\mathrm{e}}$. Figure 4 shows the axial variation of the contributions of the different terms in the stationary form of the electron energy balance (4): The power input in the magnetic filter region via $\mathbf{J}_{\mathrm{e}}$ (in particular, via the conductive flux) is in a balance with the electron energy losses in collisions (mainly inelastic collisions); the contributions of the elastic collisions as well as the energy transfer between the electrons and the dc electric field (the last term in (4)) are negligible. The formation of a minimum of the electron temperature in the filter region (Figures $\mathbf{2}$ and $\mathbf{3}$ ) stems mainly from the thermal (conductive) flux associated with diamagnetic drifts (Figure 5). According to the second term in (6), the axial (z-) decrease of $T_{\mathrm{e}}$ leads to a thermal flux in the $x$-direction (Figure 5(b)) which is related to a diamagnetic drift. This causes an increase of $T_{\mathrm{e}}$ in the $x$-direction. Consequently, the latter determines a thermal flux related again to a diamagnetic drift, however, now in the $z$-direction, that results into electron heating behind the filter and, thus, into formation of a groove in the filter region. In addition, the diamagnetic drift in the electron flux ((5) and Figure 5(a)), also contribute through the convective flux (the last term in (6))

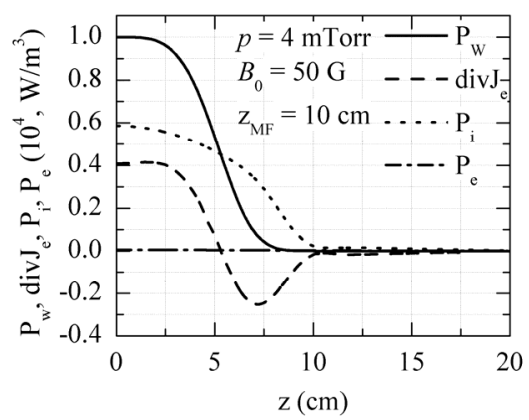

Figure 4. Axial variation (at $x=0$ ) of the contributions to the electron energy balance: $P_{w}$ (external power deposition), $\operatorname{div} \mathbf{J}_{\mathbf{e}}$ and $\mathbf{P}_{\text {coll }}=\mathbf{P}_{\text {el.coll. }}+\mathbf{P}_{\text {inel.coll. }}$ (electron energy losses in collisions presented separately for elastic $\left(P_{e}\right)$ and inelastic $\left(P_{i}\right)$ collisions); $z_{0}=10 \mathrm{~cm}, p=4 \mathrm{mTorr}$. 


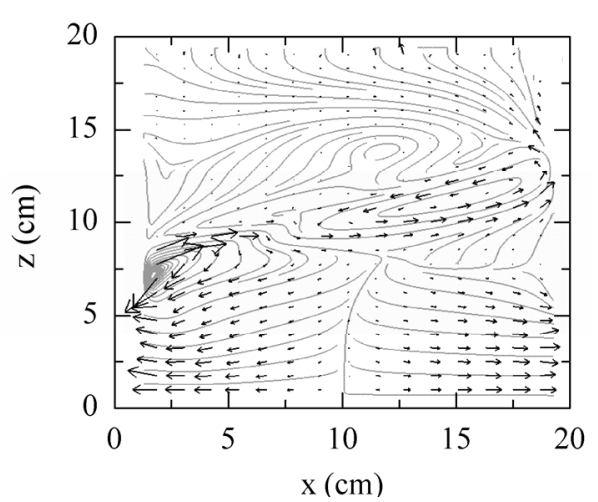

(a)



(b)

Figure 5. Arrow plot presentation of the electron flux (a) and of the electron energy flux (b) in the (x-z)-plane; $z_{0}=10 \mathrm{~cm}, p=4 \mathrm{mTorr}$.

to the increase of $T_{\mathrm{e}}$ behind the filter and, thus, to the formation of the final distribution of the electron temperature. The appearance of the maximum of $n_{\mathrm{e}}$ in the filter region is due to diffusion and thermal diffusion acting together: due to the axial drop of $T_{\mathrm{e}}$ the thermal diffusion gains in importance forming a forward thermal-diffusion flux. On the other hand, the diffusion flux is lowered, due to the magnetic field. This results into an accumulation of electrons in the filter region and a formation of the maximum of $n_{\mathrm{e}}$ there. Since only axial profiles at $x=0$ are presented in Figure 2(a) the shiftdue to the $(\mathbf{E} \times \mathbf{B})$-drift - of the maximum of $n_{\mathrm{e}}$ in the $x$-direction shown in [16], is not discussed here.

The axial variation of $T_{\mathrm{e}}$ within the full width $\Delta z_{B}$ of the magnetic filter region includes its drop, the formation of its minimum and its slight increase further on (Figure 3). Compared to the structure of the $T_{\mathrm{e}}$-profile, the structuring of the $n_{\mathrm{e}}$ - profile consists of more details. The increase of $n_{\mathrm{e}}$ in the filter region leads to formation of a minimum in front of the filter. The formation of the main maximum of $n_{\mathrm{e}}$ in the filter region is preceded by slight maximum and minimum. The strong drop of $n_{\mathrm{e}}$ after its maximum also starts within the filter region. The minimum of $T_{\mathrm{e}}$ and the maximum of $n_{\mathrm{e}}$, being slightly shifted from the center of the filter, are at the same position.

With a magnetic filter located in the vicinity of $z_{0}=15 \mathrm{~cm}$, two regions of plasma expansion without magnetic field show evidence in the axial profiles of $n_{\mathrm{e}}$ and $T_{\mathrm{e}}$ (Figures 2 and $\mathbf{3}$ ). These are the transition between the regions of the power deposition and the magnetic filter and the region behind the filter. The pure effects of plasma expansion without magnetic field are: 1) a very slight decrease of the electron temperature related to high nonlocality of the electron heating due to the high value of the thermal conductivity coefficient $\chi_{\| \mathrm{e}}$ without magnetic field (Figures 2(a) and 3(a)), and 2) strong decrease of the electron density (Figures 2(b) and 3(b)).

With a position of the magnetic filter close to $z_{0}=10$ $\mathrm{cm}$, the plasma expansion from the region of the rf power deposition is directly into the magnetic field, i.e. plasma expansion without magnetic field shows evidence only behind the filter. In this case the drop of the electron temperature in the magnetic field region starts from its value in the power deposition region.

For a position of the magnetic filter approaching $z_{0}=20 \mathrm{~cm}$, the pattern of plasma expansion without magnetic field (slight decrease of $T_{\mathrm{e}}$ and strong drop of $n_{\mathrm{e}}$ ) covers almost completely the total volume of the discharge.

Shifting the position of the filter field away from the power deposition region does not influence the axial gradient of $T_{\mathrm{e}}$ (Figure 3(a)). However, the maximum of $n_{\mathrm{e}}$ in the filter region becomes lower (Figure 3(b)), since the increase of $n_{\mathrm{e}}$ in the filter region starts from a lower value of $n_{\mathrm{e}}$. The latter is due to the larger extension of the region of plasma expansion outside the power deposition in front of the filter and, respectively, to the stronger drop of $n_{\mathrm{e}}$ there.

The structuring of the axial profiles of the electron temperature and density in the filter region as well as their complete behavior, including the region of plasma expansion before the filter (expansion outside the power deposition region) shown in Figures $\mathbf{2}$ and $\mathbf{3}$ agrees qualitatively very well with the experimental results from probe diagnostics in $[19,20]$. Not only the minimum of $T_{\mathrm{e}}$ and the maximum of $n_{\mathrm{e}}$ in the filter region have been experimentally observed. The entire behavior of the profiles, including their changes with shifting the position of the magnetic filter, follows the trends outlined in Figure 3: 1) lower $T_{\mathrm{e}}$ behind the filter and higher maximum of $n_{\mathrm{e}}$ in the filter region when the filter is located close to the power deposition region; 2) the same gradients of $T_{\mathrm{e}}$ in the filter region for different positions of the filter; 3) a minimum of $n_{\mathrm{e}}$ preceding its maximum in the filter region; and 4) the same position of the minimum of $T_{\mathrm{e}}$ and of the maximum of $n_{\mathrm{e}}$ in the filter 
region.

As it has been already mentioned, the magnetic filter is a key component in the design of the sources of negative hydrogen ions developed for fusion applications. The role of the filter to cool the electrons, necessary for local production of the negative ions by electron impact with vibrationally excited molecules, has been usually stressed on. However, the results in Figures 2(b) and 3(b) show that the structuring of the axial profile of the plasma density can be also employed in the optimization of the source. Since efficient local production of negative ions requires not only low $T_{\mathrm{e}}$ but also comparatively high $n_{\mathrm{e}}$, conditions for maximum negative ion production should be looked for by choosing a proper position of the magnetic filter with respect to the power deposition region and the extraction region.

The axial profile of the densities of the positive ions (Figure 6 where the densities of the molecular $\mathrm{H}_{2}^{+}$and $\mathrm{H}_{3}^{+}$ions are given) show the same structure as that of the electron-density profiles: strong decrease due to plasma expansion outside the power deposition region and formation of a maximum in the filter region, followed by strong drop after the filter. The structuring in the axial profile of the density of the $\mathrm{H}_{3}^{+}$-ions is better pronounced. The value of the maximum of their density in the filter region is even higher than that in the power

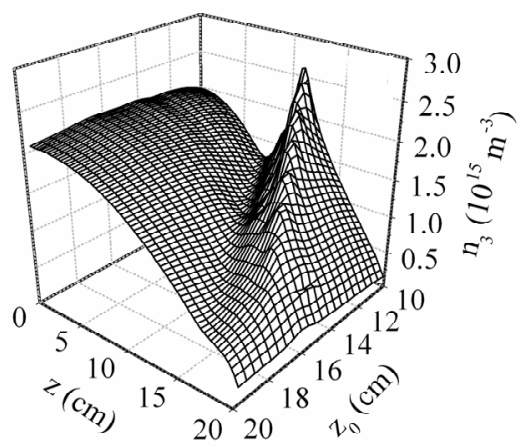

(a)

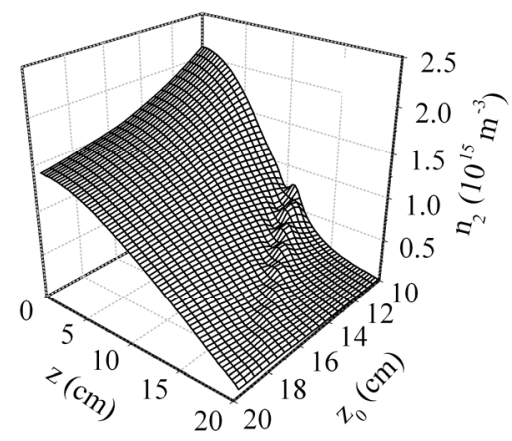

(b)

Figure 6. The same as in Figure 2 but for the densities of the $\mathrm{H}_{3}^{+}-(\mathrm{a})$ and $\mathrm{H}_{2}^{+}-(\mathrm{b})$ ions. deposition region. The changes in the axial profiles with varying the position of the filter field are also the same as of the electrons. Since the electron density is comparatively low, the concentration of the atomic $\left(\mathrm{H}^{+}\right)$ions, not shown here, is lower than that of the molecular ions (about 5 times lower). In general, the axial profiles of the ions show that the plasma quasi-neutrality in the different regions of the discharge is ensured by interplay between the concentrations of the three types of positive ions.

\subsection{Influence of the Gas Pressure}

Figure 7 shows results for the influence of the gas pressure variation on the axial discharge structure for a given position of the magnetic filter $\left(z_{0}=10 \mathrm{~cm}\right)$.

The results for the electron temperature (Figure 7(a)) and density (Figure 7(b)) in the power deposition region show the basic trends of the changes in the gas discharge behavior with varying gas pressure: higher electron temperature and lower electron density for lower gas pressure due to the increased charged particle losses via their fluxes towards the walls. Further on, in the filter region, this trend is kept: the highest $T_{\mathrm{e}}$ and the lowest $n_{\mathrm{e}}$ are for the lowest value $p=4 \mathrm{mTorr}$ of the gas pressure.

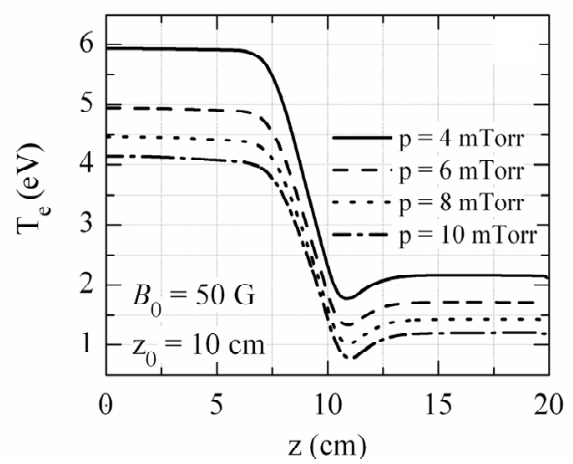

(a)

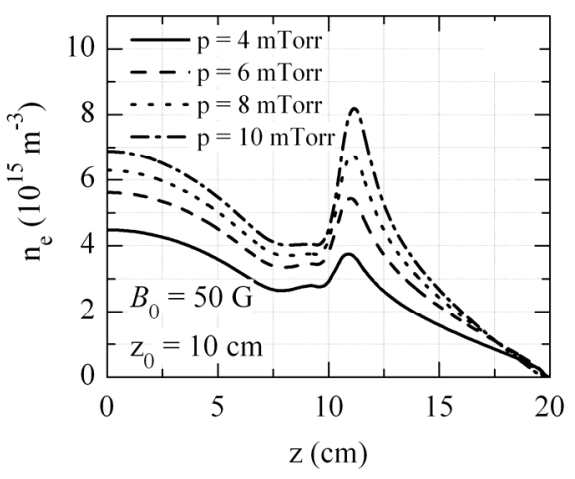

(b)

Figure 7. Comparison of the axial variation (at $x=0$ ) of the electron temperature (a) and density (b) for different values of the gas pressure $p$, as marked on the figure; $z_{0}=$ $10 \mathrm{~cm}$. 
Only at the very end of the discharge, in the wall sheath at the back wall, due to the larger gradients of $n_{\mathrm{e}}$ for higher gas pressure, the electron density drops faster when the gas pressure is higher.

The more pronounced structuring in the $n_{\mathrm{e}}(z)$-profile for higher pressure is related to the reduction - with the pressure increase - of the diffusion coefficient outside the filter region. The gradient of $T_{\mathrm{e}}$ in the filter region decreases with the gas pressure increase. The positions of the minimum of $T_{\mathrm{e}}$ and of the maximum of $n_{\mathrm{e}}$ in the filter region slightly depend on the gas pressure. The lowest value of $T_{\mathrm{e}}$ in the filter region and behind it and the highest value of $n_{\mathrm{e}}$ in the filter are for the highest value of the gas pressure.

The axial profiles of the densities of the positive ions follow the trends of the axial profiles of the electron density (Figure 7(b)). In the entire gas pressure range studied here, the concentrations of the molecular ions are higher than that of the atomic ions. Also in the entire pressure range the structuring of the density profile of the $\mathrm{H}_{3}^{+}$-ions is better pronounced and the maximum of their concentration in the filter region exceeds the density value in the power deposition region.

\subsection{Axial Profiles of the Potential of the dc Electric Field in the Discharge}

Figure 8(a) shows the axial profiles of the potential of the dc electric field for different positions $z_{0}$ of the center of the filter field for $p=4$ mTorr whereas the corresponding results obtained for a given value of $z_{0}$ $\left(z_{0}=10 \mathrm{~cm}\right)$ and varying pressure values are in Figure 8(b).

The well pronounced wall sheath at the end of the profiles ( $z$ approaching $z=20 \mathrm{~cm}$ ), with a strong drop of the dc potential there, shows discharge behavior in a free-fall regime and, thus, the necessity of accounting for the inertia terms in the momentum equations of the positive ions, as it has been done in Section 2. The minimum of $T_{\mathrm{e}}$ and the maximum of $n_{\mathrm{e}}$ in the filter region are accompanied with a change in the gradient of the potential of the dc field there, i.e. with a change in the dc electric field.

\section{Conclusion}

Based on an extension of the 2D fluid-plasma model from [16] towards description of the free-fall regime discharge maintenance, developed in the study, results for the spatial structure of low-pressure hydrogen discharges with localized rf power deposition and an external magnetic field positioned outside it are discussed. The characteristics of the remote plasma maintenance in regions without and with an external magnetic field, as determined by the nonlocality of the discharge behavior,



(a)



(b)

Figure 8. Axial profiles (at $x=0$ ) of the potential $\Phi$ of the dc electric field: (a) at a given value of the gas pressure $(p=4 \mathrm{mTorr})$ and different positions of the center $z_{0}$ of the magnetic filter, and (b) for a given value of the center of the magnetic filter $\left(z_{0}=10 \mathrm{~cm}\right)$ and different values of the gas pressure.

are outlined. Slight decrease of the electron temperature accompanied with strong drop of the electron density characterizes the plasma expansion in regions without external magnetic field whereas strong drop of the electron temperature accompanied with formation of a maximum of the electron density characterizes the plasma expansion through an external magnetic field. The analysis of the results could be employed in conclusions for optimization of the discharge as a source of volumeproduced negative ions regarding use for addional plasma heating in big fusion machines (ITER size tokamaks). Electron cooling by the magnetic filter is the result usually stressed on with regards to the requirements for local production of negative hydrogen ions. In addition, the formation of the maximum of the electron density in the filter region can be also used for reaching high density of the negative ions. For achieving this, a proper position of the magnetic filter with respect to both the power deposition region and the extraction region should be chosen.

\section{Acknowledgements}

The work is within the programme of the Bulgarian Association EURATOM/INRNE (task 2.1.1). 


\section{REFERENCES}

[1] M. J. Kushner, "Plasma Chemistry of $\mathrm{He} / \mathrm{O}_{2} / \mathrm{SiH}_{4}$ and $\mathrm{He} / \mathrm{N}_{2} \mathrm{O} / \mathrm{SiH}_{4}$ Mixtures for Remote Plasma-Activated Chemical-Vapor Deposition of Silicon Dioxide," Journal of Applied Physics, Vol. 74, No. 11, 1993, pp. 6538-6553. doi:10.1063/1.355115

[2] D. P. Lymberopoulos and D. J. Economou, "Two-Dimensional Self-Consistent Radio Frequency Plasma Simulations Relevant to the Gaseous Electronics Conference RF Reference Cell," Journal of Research of the National Institute of Standards and Technology, Vol. 100, No. 4, 1995, pp. 473-494. doi:10.6028/jres.100.036

[3] M. J. Kushner, "Hybrid Modelling of Low Temperature Plasmas for Fundamental Investigations and Equipment Design," Journal of Physics D: Applied Physics, Vol. 42, 2009, Article ID: 194013.

[4] E. Speth, H. D. Falter, P. Franzen, U. Fantz, M. Bandyopadhyay, S. Christ, A. Encheva, M. Fröschle, D. Holtum, B. Heinemann, W. Kraus, A. Lorenz, Ch. Martens, P. McNeely, S. Obermayer, R. Riedl, R. Süss, A. Tanga, R. Wilchelm and D. Wünderlich, "Overview of the RF Source Development Programme at IPP Garching," Nuclear Fusion, Vol. 46, No. 6, 2006, pp. S220-S238. doi:10.1088/0029-5515/46/6/S03

[5] Ts. V. Paunska, A. P. Shivarova, Kh. Ts. Tarnev and Ts. V. Tsankov, "2D Model of a Tandem Plasma Source: The Role of the Transport Processes," AIP Conference Proceedings, Vol. 1097, 1997, pp. 12-21.

[6] M. Bacal, "Physics Aspects of Negative Ion Sources," Nuclear Fusion, Vol. 46, No. 6, 2006, pp. S250-S259. doi:10.1088/0029-5515/46/6/S05

[7] A. J. T. Holmes, "Electron Flow through Transverse Magnetic Fields in Magnetic Multipole Arc Discharges," Review of Scientific Instruments, Vol. 53, No. 10, 1982, pp. 1517-1522. doi:10.1063/1.1136828

[8] A. J. T. Holmes, "Electron Cooling in Magnetic Multipole Arc Discharges," Review of Scientific Instruments, Vol. 53, No. 10, 1982, pp. 1523-1526. doi:10.1063/1.1136829

[9] F. A. Haas, L. M. Lea and A. J. T. Holmes, "A 'Hydrodinamic', Model of the Negatice-Ion Source," Journal of Physics D: Applied Physics, Vol. 24, No. 9, 1991, pp. 1541-1550. doi:10.1088/0022-3727/24/9/005

[10] A. J. T. Holmes, R. McAdams, G . Proudfoot, S. Cox, E. Surrey and R. King, "Intense Negative Ion Sources at Culham Laboratory," Review of Scientific Instruments, Vol. 65, No. 4, 1994, pp. 1153-1158. doi:10.1063/1.1145043

[11] M. Shirai, M. Ogasawara, T. Koishimine and A. Hatayama, "Theoretical Investigations of Electron Temperature Variation across Magnetic Filter in a Negative Ion Source," Review of Scientific Instruments, Vol. 67, No. 3, 1996, pp. 1085-1087. doi:10.1063/1.1147230

[12] A. J. T. Holmes, "A One-Dimensional Model of a Negative Ion Source," Plasma Sources Science and Technology, Vol. 5, No. 3, 1996, pp. 453-473.

doi:10.1088/0963-0252/5/3/014
[13] K. Ohi, H. Naitou, Y. Tauchi and O. Fukumasa, "Observation of the Limit Cycle in Symmetric Plasma Divided by a Magnetic Filter," Physics of Plasmas, Vol. 8, No. 1, 2001, pp. 23-30. doi:10.1063/1.1329653

[14] T. Mizuno, Y. Kitade, A. Hatayama, T. Sakurabayashi, N. Imai, T. Miroshita and T. Inoue, "Numerical Analysis of Plasma Spatial Uniformity in Negative Ion Sources by a Fluid Model," Review of Scientific Instruments, Vol. 75, No. 5, 2004, pp. 1760-1763. doi:10.1063/1.1695622

[15] H. Naitou, K. Ohi and O. Fukumasa, "Beam Instability Excited by the Magnetic Filter," Review of Scientific Instruments, Vol. 71, No. 2, 2000, pp. 875-877. doi:10.1063/1.1150318

[16] St. Kolev, St. Lishev, A. Shivarova, Kh. Tarnev and R. Wilhelm, "Magnetic Filter Operation in Hydrogen Plasmas," Plasma Physics and Controlled Fusion, Vol. 49, No. 9, 2007, pp. 1349-1369. doi:10.1088/0741-3335/49/9/001

[17] St. Kolev, G. J. M. Hagelaar and J. P. Boeuf, "Particle-inCell with Monte Carlo Collision Modeling of the Electron and Negative Hydrogen Ion Transport across a Localized Transverse Magnetic Field," Physics of Plasmas, Vol. 16, 2009, Article ID: 042318.

[18] G. J. M. Hagelaar and N. Oudini, "Plasma Transport across Magnetic Field Lines in Low-Temperature Plasma Sources," Plasma Physics and Controlled Fusion, Vol. 53, No. 12, 2011, Article ID: 124032.

[19] St. St. Lishev, A. P. Shivarova and Ts. V. Tsankov, "Experiments on the Detection of Negative Hydrogen Ions in a Small-Size Tandem Plasma Source," AIP Conference Proceedings, Vol. 1097, 2009, pp. 127-136. doi:10.1063/1.3112505

[20] St. St. Lishev and A. P. Shivarova, "Laser-Photodetachment and Faraday-Cup Measurements in the Expansion Region of a Tandem-Type Plasma Source," AIP Conference Proceedings, Vol. 1390, 2011, pp. 192-201.

[21] R. K. Janev, W. D. Langer, K. Evans Jr. and D. E. Post Jr., "Elementary Processes in Hydrogen-Helium Plasmas," Springer, Berlin, 1987.

[22] R. H. Neynaber and S. M. Trujillo, "Study of $\mathrm{H}_{2}^{+}+\mathrm{H}_{2}$ $\rightarrow \mathrm{H}_{3}{ }^{+}+\mathrm{H}$ Using Merging Beams," Physical Review, Vol. 167, No. 1, 1968, pp. 63-67. doi:10.1103/PhysRev.167.63

[23] A. Rousseau, A. Granier, G. Gousset and P. Leprince, "Microwave Discharge in $\mathrm{H}_{2}$ : Influence of H-Atom Density on the Power Balance," Journal of Physics D: Applied Physics, Vol. 27, No. 7, 1994, pp. 1412-1422. doi:10.1088/0022-3727/27/7/012

[24] I. Koleva, Ts. Paunska, H. Schlüter, A. Shivarova and Kh. Tarnev, "Surface-Wave Produced Discharges in Hydrogen: I. Self-Consistent Model of Diffusion-Controlled Discharges," Plasma Sources Science and Technology, Vol. 12, No. 4, 2003, pp. 597-607. doi:10.1088/0963-0252/12/4/311

[25] St. Lishev, A. Shivarova and Kh. Tarnev, "On the Inertia Term in the Momentum Equation in the Free-Fall Regime of Discharge Maintenance," Journal of Plasma Physics, 
Vol. 77, No. 4, 2011, pp. 469-478.

doi:10.1017/S0022377810000620

[26] S. Weissman and E. A. Mason, "Estimation of the Mutual Diffusion Coefficient of Hydrogen Atoms and Mole- cules," Journal of Chemical Physics, Vol. 36, No. 3, 1962, pp. 794-797. doi:10.1063/1.1732612 